\title{
Dark Matter Searches with Germanium Detectors with sub-keV Sensitivities
}

\author{
Henry T. Wong \\ Institute of Physics, Academia Sinica \\ 128 Section 2, Academia Road, NanKang, Taipei 11529, Taiwan \\ E-mail: htwongaphys.sinica.edu.tw
}

\author{
Shin-Ted Lin ${ }^{1}$ (for TEXONO + CDEX Collaboration) \\ Institute of Physics, Academia Sinica \\ 128 Section 2, Academia Road, NanKang, Taipei 11529, Taiwan \\ E-mail: Iinstaphys.sinica.edu.tw
}

\begin{abstract}
The theme of the TEXONO research program is on the studies of low energy neutrino and dark matter physics. The current goals of the TEXONO research program are on the development of germanium detectors with sub-keV sensitivities to realize experiments on neutrino magnetic moments, neutrino-nucleus coherent scattering, as well as WIMP dark matter searches. The compatible sensitivities at low mass WIMP searches has achieved with a four-channel ultra-lowenergy germanium prototype detector each with an active mass of $5 \mathrm{~g}$ at the Kuo-Sheng Neutrino Laboratory (KSNL). Data are being taken with a $500 \mathrm{~g}$ Point Contact Germanium detector will be demonstrated. The projected sensitivities for WIMP and axion dark matter searches would be also discussed. The dark matter program evolves into a dedicated experiment with CDEX at China Jing-Ping underground laboratory (CJPL) in Sichuan, China.
\end{abstract}

35th International Conference of High Energy Physics (ICHEP2010)

Paris, France

July 22-28, 2010

Speaker 


\section{Introduction}

A research program on low energy neutrino and dark matter physics is pursued at the KuoSheng Neutrino Laboratory (KSNL) by the TEXONO Collaboration [1]. The laboratory is located at a distance of $28 \mathrm{~m}$ from a $2.9 \mathrm{GW}$ reactor core and has an overburden of about 30 meter-water-equivalent. Results on neutrino magnetic moments [2] and neutrino-electron scattering cross-section have been obtained [3].The present goals are to develop advanced detectors with $\mathrm{kg}$-size target mass, $100 \mathrm{eV}$ range threshold and low-background specifications [4] for the searches of Weakly Interacting Massive Particles (WIMPs) at the low-mass region as well as the studies of neutrino-nucleus coherent scattering [5] and neutrino magnetic moments.

\section{Performance of sub-keV Germanium Detectors}

The design of Point-Contact Germanium (PCGe) detectors was first proposed in the 1980's [6], offering the merits of sub-keV sensitivities with kg-scale target mass. The efficiency factors below the electronic noise edge adopted to similar procedures [5] to those developed for the $4 \times 5$ g Ultra-Low-Energy Germanium (ULEGe) prototype detector.

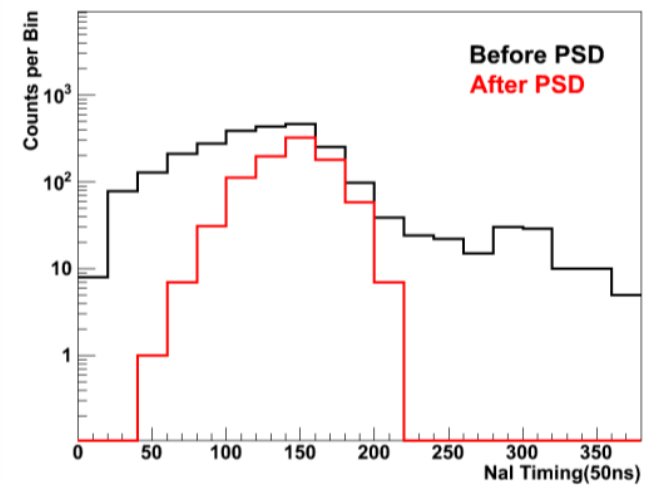

Figure 1: Events as a function of relative timing between $\mathrm{ACV}-\mathrm{NaI}(\mathrm{Tl})$ and $\mathrm{PCGe}$ systems, before and after PSD selection.

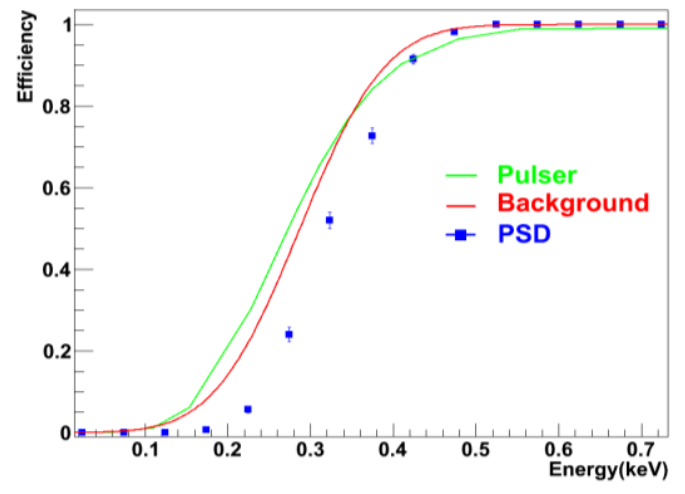

Figure 2: The trigger and analysis efficiencies of the $500 \mathrm{~g}$ PCGe detector, as derived from the The test pulser and in situ, respectively.

The relative timing between the PCGe and anti-Compton (ACV) NaI(Tl) detectors is shown in Figure 1, for "sub-noise edge" events at 200-400 eV before and after the pulse shape discrimination (PSD) selection processes. The trigger efficiencies were measured with two methods. The fractions of calibrated pulser events above the discriminator threshold provided the first measurement, while the studies on the amplitude distributions of in situ data contributed to the other. Events in coincidence with ACV at the "50-200 ns" window are due to multiple Compton scatterings, which are actual physical processes having similar pulse shapes as the neutrino and WIMP signals. It can be seen that only these events have substantial probabilities of surviving the cuts, and the fractions constitute to the PSD efficiencies. The threshold at $50 \%$ combined efficiencies is $\sim 350 \mathrm{eV}$. 


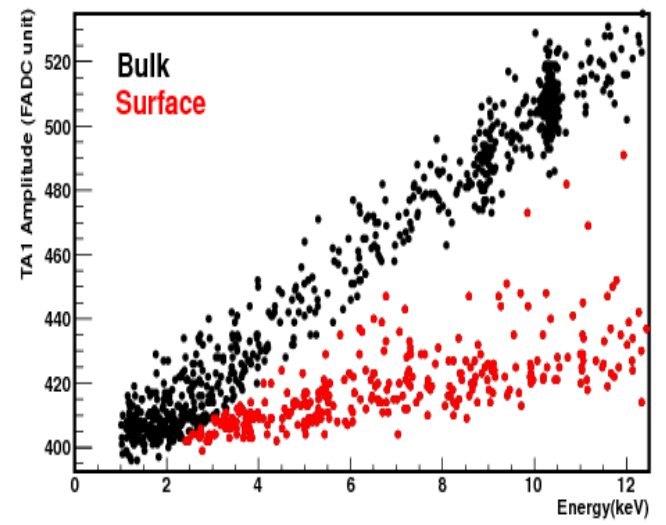

Figure 3: The separation of surface and bulk events down to $2 \mathrm{keV}$. The $\mathrm{X}$-ray peaks are located in the bulk region.

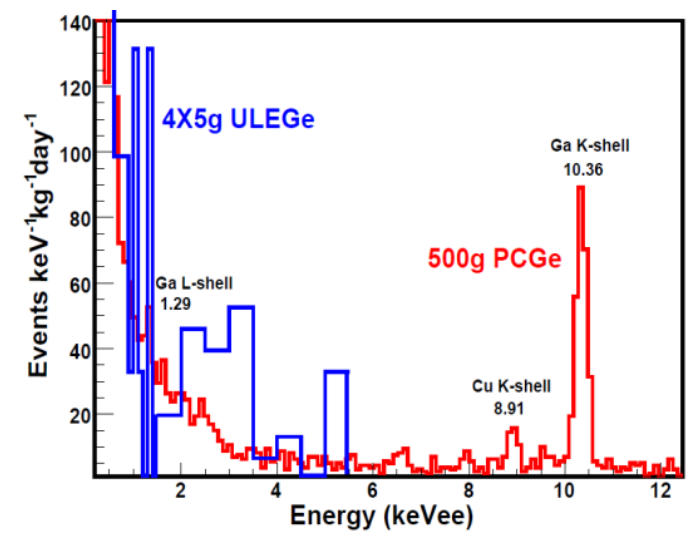

Figure 4: The measured spectra of ULEGe and PCGe after various background suppression procedures.

The difference of surface and bulk of PCGe down to $2 \mathrm{keV}$ can be distinguished with fast pulse shapes of Timing Amplifier through low pass filter method at $200 \mathrm{MHz}$ FADC. The redefined amplitude as a function of energy is shown in Figure 3. The surface signals reduce due to the "inactive layer" is not totally dead.

\section{Dark matter searches}

A four-channel ULEGe prototype detector with a total active mass of $20 \mathrm{~g}$ [5] and a compact PCGe with a $500 \mathrm{~g}$ have collected low-background data at KSNL. The spectra with $0.34 \mathrm{~kg}$-day of exposure for ULEGe and $15.8 \mathrm{~kg}$-day for PCGe is displayed in Figure 4.

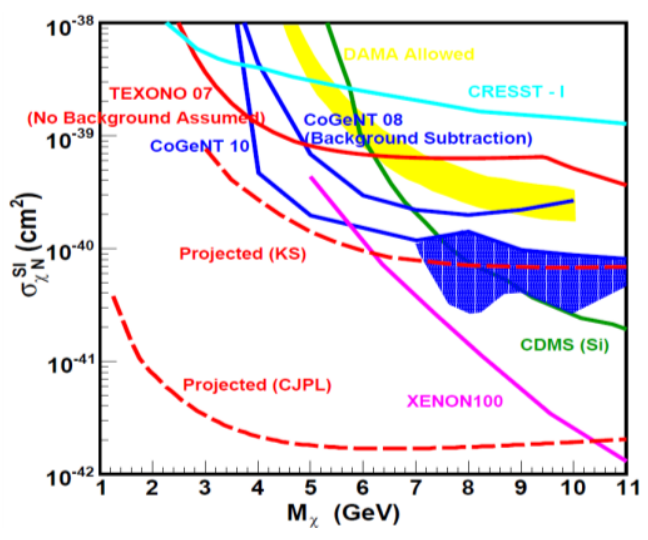

Figure 5: Exclusion plot of the spinindependent $\chi \mathrm{N}$ cross section versus WIMP Mass. The projected sensitivities of KSNL and CJPL are also illustrated.

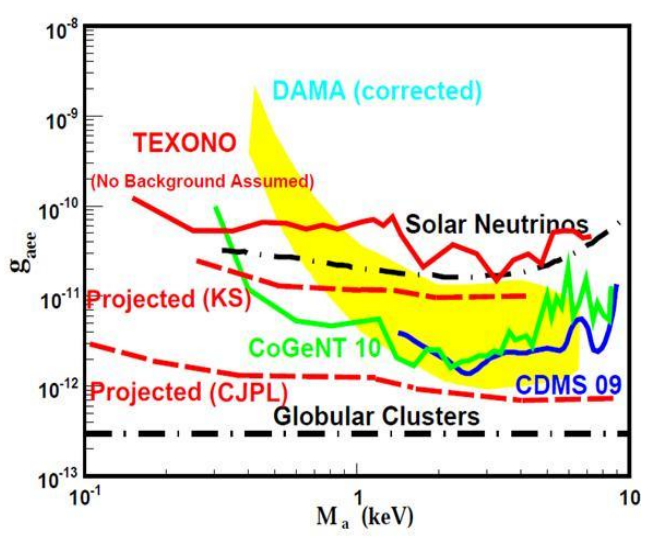

Figure 6: The axion-electron coupling versus axion mass exclusion plot of TEXONO together with other experiments at $90 \%$ C. L.. 
Generic gamma-like sources and residues of cosmic-induced, as well as surface events below $2 \mathrm{keV}$ are studied for background understanding and subtraction. The projected sensitivities are obtained by subtracting known background for KSNL. On the other hand, for CJPL it is obtained by assuming 1 cpkkd of background level with $1 \mathrm{~kg}$-year data volume. Intensive background and optimization studies with the PCGe at KSNL are underway.

Constraints on WIMP-nucleon spin-independent couplings as a function of WIMP-mass were derived and the results are illustrated in Figure 5. Overlaid on the plots are results from experiments which define the current exclusion boundaries and the DAMA-allowed regions [7, 8]. TEXONO exclusion plots for axion-electron coupling versus axion mass together with other experiments [8] are shown in Figure 6.

\section{Status and Plans}

An excellent candidate site for a deep underground laboratory was recently identified in Sichuan, China where the China Jin-Ping Laboratory (CJPL) is being constructed [9]. The laboratory has more than $2500 \mathrm{~m}$ of rock overburden, is accessible by a road tunnel built for public traffic, and is supported by excellent infrastructures already available near the entrance. The construction of the first cavern of size $6 \mathrm{~m}$ (height) $\times 6 \mathrm{~m}$ (width) $\times 40 \mathrm{~m}$ (depth) has been completed recently.

The low energy neutrino physics program will proceed at KSNL with a 900 g PCGe detector. In parallel, dedicated dark matter search will be conducted at CJPL as a first experimental program of TEXONO-CDEX collaboration commencing in 2010 with both $20 \mathrm{~g}$ ULEGe and $500 \mathrm{~g}$ PCGe detectors.

\section{References}

[1] H. T. Wong, Mod. Phys. Lett. A 19 (1207) 2004.

[2] H. B. Li et al., Phys. Rev. Lett. 90 (131802) 2003; H. T. Wong et al., Phys. Rev. D 75 (012001) 2007.

[3] M. Deniz et al., Phys. Rev. D 81 (072001) 2009; M. Deniz et al, Phys. Rev. D 82 (033004) 2010.

[4] H. T. Wong, Mod. Phys. Lett. A 19 (1207) 2004.

[5] S. T. Lin et al., Phys. Rev. D 76 (061101 R) 2009 and reference therein.

[6] P. N. Luke et al., IEEE Trans. Nuc. Sci. 36 (926) 1989.

[7] E. Aprile, et al., [astro-ph/1005.0380].

[8] C.E. Aalset et al., [astro-ph/1002.4703v2].

[9] D. Normile, Science 324 (1246) 2009. 